\title{
CONGRUENCE LATTICES OF FINITE SEMIMODULAR LATTICES
}

\author{
G. GRÄTZER, H. LAKSER, AND E. T. SCHMIDT
}

\begin{abstract}
We prove that every finite distributive lattice can be represented as the congruence lattice of a finite (planar) semimodular lattice.
\end{abstract}

\section{INTRODUCTION}

A classical result of R. P. Dilworth (circa 1940, unpublished, see II, pp. 455457 ) states that a finite distributive lattice $D$ can be represented as the congruence lattice of a finite lattice $L$.

There are a number of papers strengthening this result by requiring that the lattice $L$ representing $D$ have special properties. The lattice $L$ constructed by Dilworth is atomistic. A sectionally complemented lattice $L$ is constructed in G. Grätzer and E. T. Schmidt 7, while a planar lattice is constructed in G. Grätzer and H. Lakser 4. A "small" lattice $L$ is constructed in G. Grätzer, H. Lakser, and E. T. Schmidt 5 : if $D$ has $n$ join-irreducible elements, the lattice $L$ is of size $O\left(n^{2}\right)$. (This is "best possible", according to G. Grätzer, I. Rival, and N. Zaguia 6.)

In this paper, we construct a semimodular lattice $L$ :

Theorem. Every finite distributive lattice $D$ can be represented as the congruence lattice of a finite semimodular lattice $S$. In fact, $S$ can be constructed as a planar lattice of size $O\left(n^{3}\right)$, where $n$ is the number of join-irreducible elements of $D$.

This result, with size $O\left(n^{4}\right)$, was announced in 9 ; the present paper contains an improved construction, due to the second author, yielding size $O\left(n^{3}\right)$. It would be interesting to decide whether the size $O\left(n^{2}\right)$ is possible for (planar) semimodular lattices.

\section{Preliminaries}

We use the basic concepts and notations as in 2 ; in particular, for a finite distributive lattice $D, \mathrm{~J}(D)$ denotes the poset of join-irreducible elements. Con $L$ denotes the congruence lattice of the lattice $L$. For a prime interval $\mathfrak{p}=[a, b]$, $\Theta(\mathfrak{p})=\Theta(a, b)$ is the smallest congruence collapsing $a$ and $b . \mathfrak{C}_{2}$ denotes the twoelement chain.

It is convenient to describe congruences of a finite lattice using coloring:

Date: Dec. 12, 1996.

1991 Mathematics Subject Classification. Primary 06B10; Secondary 08A05.

Key words and phrases. Congruence lattice, semimodular, planar, finite.

The research of the first and second authors was supported by the NSERC of Canada.

The research of the third author was supported by the Hungarian National Foundation for Scientific Research, under Grant No. T7442. 


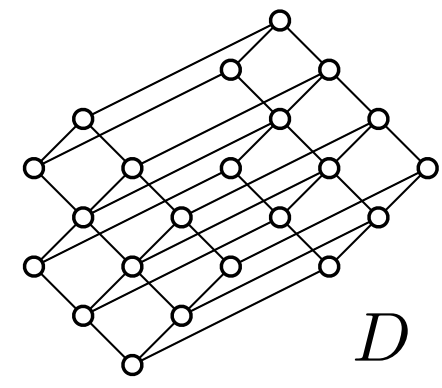

Figure 1

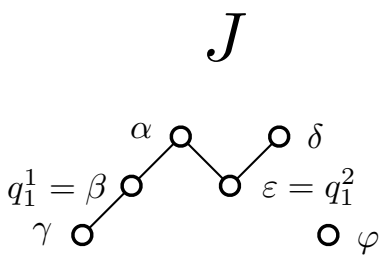

Figure 2

Let $L$ be a finite lattice and let $\Gamma$ be a finite set; the elements of $\Gamma$ will be called colors. A coloring $\mu$ of $L$ over $\Gamma$ is a map

$$
\mu: \mathfrak{P}(L) \rightarrow \Gamma
$$

of the set of prime intervals $\mathfrak{P}(L)$ of $L$ into $\Gamma$ satisfying the condition: if two prime intervals generate the same congruence relation of $L$, then they have the same color; that is,

$$
\mathfrak{p}, \mathfrak{q} \in \mathfrak{P}(L) \text { and } \Theta(\mathfrak{p})=\Theta(\mathfrak{q}) \quad \text { imply that } \quad \mathfrak{p} \mu=\mathfrak{q} \mu \text {. }
$$

Since the join-irreducible congruences of $L$ are exactly those that can be generated by prime intervals, equivalently, $\mu$ can be regarded as a map of the set $\mathrm{J}(\mathrm{Con} L)$ of join-irreducible congruences of $L$ into $\Gamma$ :

$$
\mu: \mathrm{J}(\operatorname{Con} L) \rightarrow \Gamma \text {. }
$$

In view of this condition, it is enough to define $\mu$ on sufficiently many prime intervals so that every prime interval is projective to one on which $\mu$ is defined.

Let $A$ and $B$ be lattices, $D_{A}$ a dual ideal of $A, I_{B}$ an ideal of $B$, and $D_{B}$ a dual ideal of $B$. Let us assume that $D_{A}, I_{B}$, and $D_{B}$ are isomorphic. We now define what it means that we obtain $C$ by gluing $B$ to $A k$-times. For $k=1$, let $C$ be the gluing of $A$ and $B$ over $D_{A}$ and $I_{B}$ with the dual ideal $D_{B}$ regarded as a dual ideal $D_{C}$ of $C$. Now if $C_{k-1}$ with the dual ideal $D_{C_{k-1}}$ is the gluing of $B$ to $A$ $k$-1-times, then we glue $C_{k-1}$ and $B$ over $D_{C_{k-1}}$ and $I_{B}$ to obtain $C$ the gluing of $B$ to $A k$-times with the dual ideal $D_{B}$ regarded as a dual ideal $D_{C}$ of $C$. Observe that if $A$ and $B$ are semimodular, then so is $C$. Since we construct the lattice $S$ of the Theorem from semimodular components using gluing, the semimodularity of $S$ follows.

\section{The CONSTRUCTION}

We construct the semimodular lattice $S$ of the Theorem in several steps. The construction is easy to follow on pictures but somewhat notational in a formal presentation. So we suggest that the reader follow it on the example we present; the example is the smallest one that illustrates various aspects of the construction. This example represents the 22-element distributive lattice $D$ of Figure 1 as the congruence lattice of a semimodular lattice. The poset $J$ of join-irreducibles has six elements, and it is shown in Figure 2.

Take the eight-element, nonmodular, semimodular lattice $S_{8}$ of Figure 3. $S_{8}$ has an ideal, $I_{S_{8}}=(b]$, and a dual ideal, $D_{S_{8}}=\left[c\right.$ ), both isomorphic to $\mathfrak{C}_{2}$; we shall utilize these for repeated gluings. The elements of $I_{S_{8}}$ are black filled and the 


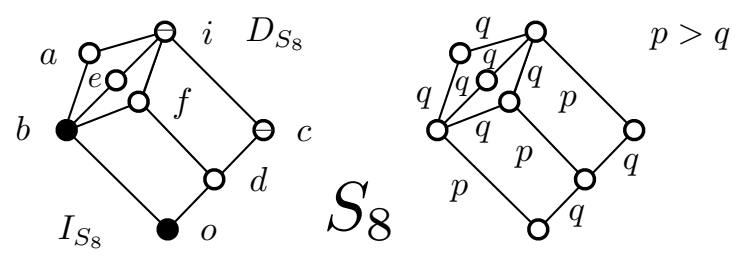

Figure 3

elements of $D_{S_{8}}$ are striped on Figure 3. It is easy to see that the congruence lattice of $S_{8}$ is the three-element chain. Using the notation $\mathrm{J}\left(\mathrm{Con} \mathfrak{C}_{3}\right)=\{p, q\}$, with $p>q$, we also show the colored $S_{8}$ in Figure 1 .

Let $D$ be a finite distributive lattice, and let $J=\mathrm{J}(D)$ be the poset of its join-irreducible elements, $n=|J|$. We enumerate

$$
p_{1}, p_{2}, \ldots, p_{m}
$$

the non-minimal elements of $J$. For every $p_{i}, i=1,2, \ldots, m$, let

$$
v\left(p_{i}\right)=\left\{q_{i}^{1}, q_{i}^{2}, \ldots, q_{i}^{k_{i}}\right\}
$$

denote the set of all lower covers of $p_{i}$ in $J$; since $p_{i}$ is non-minimal, it follows that $k_{i}>0$. Let

$$
r_{1}, r_{2}, \ldots, r_{t}
$$

enumerate all elements of $J$ that are incomparable with all other elements.

In the example, $m=3, t=1$. Let

$$
\begin{array}{ll}
p_{1}=\alpha, & v(\alpha)=\{\beta, \varepsilon\}, \quad q_{1}^{1}=\beta, \quad q_{1}^{2}=\varepsilon, \\
p_{2}=\beta, & v(\beta)=\{\gamma\}, \\
p_{3}=\delta, & v(\delta)=\{\varepsilon\} .
\end{array}
$$

So $k_{1}=2, k_{2}=k_{3}=1$.

\section{Step 1.}

For every $i$, with $1 \leq i \leq m$, we construct a lattice $A_{i}$ with an ideal $I_{i}$ and a dual ideal $D_{i}$, where $I_{i}$ is a chain of length $2\left(k_{i}+\cdots+k_{m}\right)$ and $D_{i}$ is a chain of length $2\left(k_{i+1}+\cdots+k_{m}\right)$.

Now we shall use twice the construction, gluing $k$-times, described in Section $\boldsymbol{D}$ To form $A_{i}$, glue $S_{8}$ to itself $\left(k_{i}-1\right)$-times with the ideal $I_{S_{8}}$ and the dual ideal $D_{S_{8}}$, to obtain the lattice $A_{i}^{1}$ with a dual ideal $D_{A_{i}^{1}}$. Now take

$$
\mathfrak{C}_{2}^{2}=\{\langle 0,0\rangle,\langle 0,1\rangle,\langle 1,0\rangle,\langle 1,1\rangle\}
$$

with the ideal

$$
I_{\mathfrak{C}_{2}^{2}}=\{\langle 0,0\rangle,\langle 1,0\rangle\}
$$

and the dual ideal

$$
D_{\mathfrak{C}_{2}^{2}}=\{\langle 0,1\rangle,\langle 1,1\rangle\},
$$

and glue $2\left(k_{i+1}+\cdots+k_{m}\right)$-times $\mathfrak{C}_{2}^{2}$ to $A_{i}^{1}$. The ideal $I_{i}$ is generated by the element $\langle 0,1\rangle$ of the top $\mathfrak{C}_{2}^{2}$, while $D_{i}$ is generated by the unit element of $A_{i}^{1}$.

We define a coloring $\mu_{i}$ of $A_{i}$ as follows. On any copy of $S_{8},[o, b] \mu_{i}=p_{i}$ and on the $j$-th copy of $S_{8}$,

$$
[o, d] \mu_{i}=[d, c] \mu_{i}=q_{i}^{j}
$$




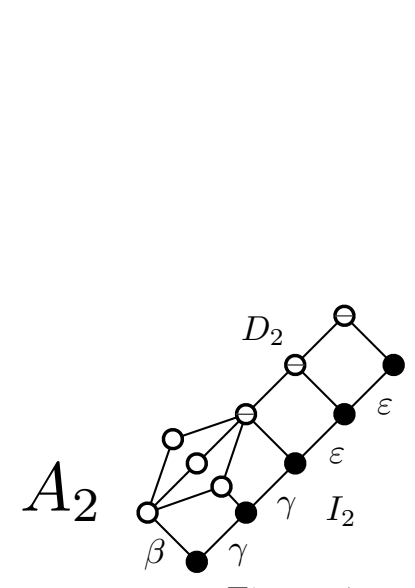

Figure 4

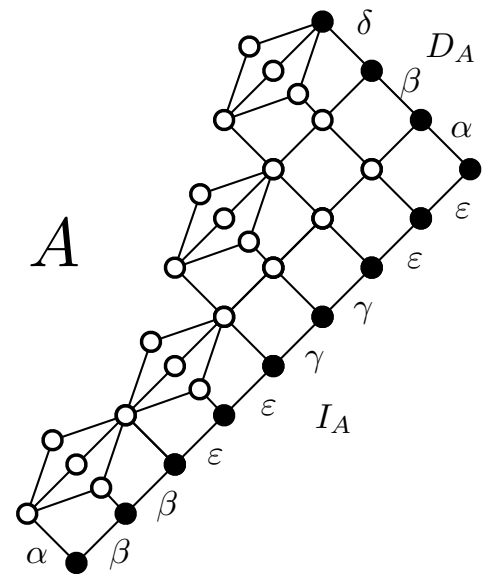

Figure 5

on the first two copies of $\mathfrak{C}_{2}^{2}$,

$$
[\langle 0,1\rangle,\langle 1,1\rangle] \mu_{i}=q_{i+1}^{1},
$$

on the next two copies,

$$
[\langle 0,1\rangle,\langle 1,1\rangle] \mu_{i}=q_{i+1}^{2},
$$

after $k_{i+1}$ pairs, the next two satisfies

$$
[\langle 0,1\rangle,\langle 1,1\rangle] \mu_{i}=q_{i+2}^{1},
$$

and so on.

Figure 4 shows $A_{2}$ for the example. The elements forming $I_{2}$ are black filled; the elements forming $D_{2}$ are striped. Note that $I_{2}$ is of length $2\left(k_{2}+k_{3}\right)=4$, while $D_{2}$ is of length $2 k_{3}=2$.

Lemma 1. $\mu_{i}$ is a coloring of $A_{i}$. The join-irreducible congruences of $A_{i}$ are generated by prime intervals of $I_{i}$ and by $[o, b]$ of the bottom $S_{8}$ in $A_{i}$. If $\mathfrak{p}$ and $\mathfrak{q}$ are $[o, b]$ or a prime interval $[o, d]$ or $[d, c]$ of a copy of $S_{8}$ in $A_{i}$, then $\Theta(\mathfrak{p}) \geq \Theta(\mathfrak{q})$ iff $\mathfrak{p} \mu_{i} \geq \mathfrak{q} \mu_{i}$. In particular, $\Theta(o, b) \succ \Theta(o, d)$ in $\mathrm{J}\left(\operatorname{Con} A_{i}\right)$, where $o, b, d$ are in $a$ copy of $S_{8}$ in $A_{i}$. If $\mathfrak{p}$ is a prime interval $[\langle 0,1\rangle,\langle 1,1\rangle]$ in a copy of $\mathfrak{C}_{2}^{2}$, then $\Theta(\mathfrak{p})$ is incomparable to any $\Theta(\mathfrak{q})$, where $\mathfrak{q}$ is $[o, b]$ or a prime interval of $I_{i}$ different from $\mathfrak{p}$.

Proof. This is trivial since every prime interval of $S_{8}$ is projective to one of $[o, b]$, $[o, d],[d, c]$.

\section{Step 2.}

We define the lattice $A$ by gluing together the (colored) lattices $A_{i}, 1 \leq i \leq m$.

For $1 \leq i \leq m$, we define, by induction, the lattice $\bar{A}_{i}$, which contains $A_{i}$, and, therefore, $D_{i}$, as a dual ideal. Let $\bar{A}_{1}=A_{1}$. Assume that $\bar{A}_{i}$ with $D_{i}$ as a dual ideal has been defined. Observe that both $D_{i}$ and $I_{i+1}$ are chains of length $2\left(k_{i+1}+\cdots+k_{m}\right)$, and so they are isomorphic; in fact, this isomorphism preserves colors. We glue $\bar{A}_{i}$ to $A_{i+1}$ over $D_{i}$ and $I_{i+1}$ to obtain $\bar{A}_{i+1}$. Define $A=\bar{A}_{m}$ and $I_{A}=I_{1}$.

Observe that $\mu_{i}$ on $D_{i}$ agrees with $\mu_{i+1}$ on $I_{i+1}$; therefore, the $\mu_{i}, 1 \leq i \leq m$, define a coloring $\mu_{A}$ of $A$. 
Let $D_{A}$ be the dual ideal of $A$ generated by the element $\langle 0,1\rangle$ of the top $\mathfrak{C}_{2}^{2}$ in $A_{1} . D_{A}$ is a chain of length $m$. The prime interval $[o, b]$ in the bottom $S_{8}$ in $A_{i}$ $(1 \leq i \leq m)$ is projective to a unique prime interval $\mathfrak{p}$ of $D_{A}$; define $\mathfrak{p} \mu_{A}=[o, b] \mu_{A}$.

Figure 5 show this lattice for the example. The elements of $I_{A}$ and $D_{A}$ are black filled.

Lemma 2. $\mu_{A}$ is a coloring of $A$. The join-irreducible congruences of $A$ are generated by prime intervals of $I_{A}$ and $D_{A}$. Let $\mathfrak{p}$ and $\mathfrak{q}$ be prime intervals in $I_{A}$ and $D_{A}$.

(i) If $\mathfrak{p}$ and $\mathfrak{q}$ are prime intervals of $D_{A}$, then $\Theta(\mathfrak{p})$ and $\Theta(\mathfrak{q})$ are incomparable.

(ii) If $\mathfrak{p}$ is a prime interval of $D_{A}$ and $\mathfrak{q}$ is a prime interval of $I_{A}$, then $\Theta(\mathfrak{p})$ and $\Theta(\mathfrak{q})$ are comparable iff $\mathfrak{p} \subseteq A_{i}$, for some $1 \leq i \leq m, \mathfrak{q}$ is perspective to some $[o, d]$ or $[d, c]$ in some $S_{8}$ in $A_{i}$; in which case, $\Theta(\mathfrak{p}) \succ \Theta(\mathfrak{q})$ in $\mathrm{J}(\operatorname{Con} A)$.

(iii) If $\mathfrak{p}$ and $\mathfrak{q}$ are prime intervals of $I_{A}$, then $\Theta(\mathfrak{p}) \geq \Theta(\mathfrak{q})$ iff $\mathfrak{p}$ and $\mathfrak{q}$ are perspective to prime intervals $\mathfrak{p}^{\prime}$ and $\mathfrak{q}^{\prime}$ in some $A_{i}$, respectively, for some $1 \leq i \leq m$, and $\mathfrak{p}^{\prime}$ and $\mathfrak{q}^{\prime}$ are adjacent edges of some $S_{8}$ in $A_{i}$; in which case, $\Theta(\mathfrak{p})=\Theta(\mathfrak{q})$.

Proof. This is obvious from the statement that if $A$ and $B$ are glued together over the dual ideal $D$ of $A$ and the ideal $I$ of $B$, then a congruence of the glued lattice is obtained from a congruence $\Theta$ of $A$ and a congruence $\Phi$ of $B$ with the property that the restriction of $\Theta$ to $D$ agrees with the restriction of $\Phi$ to $I$.

Observe that the congruence lattice of $A$ is still quite different from $D$ in two ways: the congruences that correspond to the $r_{i}$ are still missing; prime intervals in $I_{A} \cup D_{A}$ of the same color generate incomparable congruences with one exception: they are adjacent intervals in $I_{A}$, perspective to the two prime intervals of some $S_{8}$ in some $A_{i}$. For instance, in the example, see Figure 5 , the prime interval of $D_{A}$ of color $\beta$ generates a congruence incomparable to the congruence generated by a prime interval of $I_{A}$ of color $\beta$; also, a prime interval of color $\varepsilon$ in the top part of $I_{A}$ generates a congruence incomparable to the congruence generated by a prime interval of color $\varepsilon$ in the lower part of $I_{A}$.

\section{Step 3.}

We extend $A$ to a lattice $B$ with an ideal $I_{B}$ which is a chain and which has the property that every prime interval of $B$ is projective to a prime interval of $I_{B}$.

This step is easy. We form the lattice $D_{A}^{2}$ with the ideal

$$
I_{D_{A}^{2}}=\left\{\left\langle x, 0_{D_{A}}\right\rangle \mid x \in D_{A}\right\},
$$

where $0_{D_{A}}$ is the zero of $D_{A}$. Let $1_{D_{A}}$ denote the unit element of $D_{A}$ and, for $x \in D_{A}, x<1_{D_{A}}$, let $x^{*}$ denote the cover of $x$ in $D_{A}$. For every $x \in D_{A}, x<1_{D_{A}}$, we add an element $m_{x}$ to $D_{A}^{2}$ so that the elements

$$
\langle x, x\rangle,\left\langle x, x^{*}\right\rangle,\left\langle x^{*}, x\right\rangle, x_{m},\left\langle x^{*}, x^{*}\right\rangle
$$

form a sublattice isomorphic to $\mathfrak{M}_{3}$ with $\langle x, x\rangle$ as zero and $\left\langle x^{*}, x^{*}\right\rangle$ as unit. Let $M$ be the resulting lattice. Obviously, $M$ is a finite planar modular lattice whose congruence lattice is isomorphic to the congruence lattice of $D_{A} . I_{D_{A}^{2}}$ is also an ideal of $M$; we shall denote it by $I_{M}$.

Figure 6 shows $M$ for the example. The elements of $I_{M}$ are black filled.

We glue $A$ to $M$ over $D_{A}$ and $I_{M}$ to obtain $B$. Let $I_{B}$ be defined as the ideal generated by $\left\langle 0,1_{D_{A}}\right\rangle$. We define $\mu_{B}$ as an extension of $\mu_{A}$; every prime interval $\mathfrak{p}$ of $M$ is projective to exactly one prime interval $\overline{\mathfrak{p}}$ of $I_{M}$, we define $\mathfrak{p} \mu_{B}=\overline{\mathfrak{p}} \mu_{A}$. 


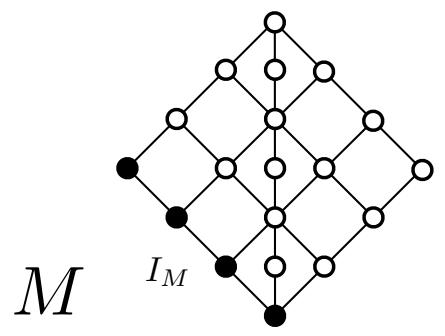

Figure 6

Lemma 3. $\mu_{B}$ is a coloring of $B$. The join-irreducible congruences of $B$ are generated by prime intervals of $I_{B}$. Let $\mathfrak{p}$ and $\mathfrak{q}$ be prime intervals in $I_{B}$.

(i) If $\mathfrak{p}$ and $\mathfrak{q}$ are prime intervals of $M$, then $\Theta(\mathfrak{p})$ and $\Theta(\mathfrak{q})$ are incomparable.

(ii) If $\mathfrak{p}$ is a prime interval of $M$ and $\mathfrak{q}$ is a prime interval of $I_{A}$, then $\Theta(\mathfrak{p})$ and $\Theta(\mathfrak{q})$ are related exactly as $\Theta_{A}(\overline{\mathfrak{p}})$ and $\Theta_{A}(\mathfrak{q})$ are related in $A$.

(iii) If $\mathfrak{p}$ and $\mathfrak{q}$ are prime intervals of $I_{A}$, then $\Theta(\mathfrak{p})$ and $\Theta(\mathfrak{q})$ are related exactly as $\Theta_{A}(\mathfrak{p})$ and $\Theta_{A}(\mathfrak{q})$ are related in $A$.

Proof. This is obvious from the congruence structure of $M$.

\section{Step 4.}

We extend $B$ to the lattice $S$ of the Theorem.

This is also an easy step. We take a chain $C$ of length $n$ and we color $C$ over $J$ so that the coloring is a bijection. We form the lattice $C \times I_{B}$. For every pair of prime intervals, $\mathfrak{p}=[a, b]$ of $C$ and $\mathfrak{q}=[c, d]$ of $I_{B}$, if $\mathfrak{p}$ and $\mathfrak{q}$ have the same color, then we add an element $m(\mathfrak{p}, \mathfrak{q})$ to $C$ over $J$ so that the elements

$$
\langle a, c\rangle,\langle b, c\rangle,\langle a, d\rangle, m(\mathfrak{p}, \mathfrak{q}),\langle b, d\rangle
$$

form a sublattice isomorphic to $\mathfrak{M}_{3}$. Let $N$ denote the resulting lattice. $N$ is obviously modular and planar. Set

$$
\begin{aligned}
I_{N} & =\left\{\left\langle x, 0_{I_{B}}\right\rangle \mid x \in C\right\}, \\
D_{N} & =\left\{\left\langle 1_{C}, x\right\rangle \mid x \in I_{B}\right\},
\end{aligned}
$$

where $0_{I_{B}}$ is the zero of $I_{B}$ and $1_{C}$ is the unit of $C$. Then $I_{N}$ is the ideal of $N$ (isomorphic to $C$ ) and $D_{N}$ is a dual ideal of $N$ (isomorphic to $I_{B}$ ). Every prime interval of $N$ is projective to a prime interval of $I_{N}$, so we have a natural coloring $\mu_{N}$ on $N$. Note that this coloring agrees with the coloring $\mu_{B}$ on $D_{N}$ under the isomorphism with $I_{B}$.

We glue $N$ to $B$ over $D_{N}$ and $I_{B}$ to obtain $S$ with the coloring $\mu_{S}$. Set $I_{S}=I_{N}$. Figure 7 is a sketch of $S$.

It is clear from the construction and from the lemmas that every prime interval of $S$ is projective to a prime interval of $I_{S}$ and that distinct prime intervals of $I_{S}$ generate distinct join-irreducible congruences of $S$.

It remains to see that if $\mathfrak{p}$ and $\mathfrak{q}$ are distinct prime intervals, then $\Theta(\mathfrak{p}) \geq \Theta(\mathfrak{q})$ iff $\mathfrak{p} \mu_{S} \geq \mathfrak{q} \mu_{S}$. Since $J$ is finite, it is sufficient to prove that $\Theta(\mathfrak{p}) \succ \Theta(\mathfrak{q})$ in $\mathrm{J}(\operatorname{Con} S)$ iff $\mathfrak{p} \mu_{S} \succ \mathfrak{q} \mu_{S}$ in $\mathrm{J}(D)$. But this is clear since if $\mathfrak{p} \mu_{S} \succ \mathfrak{q} \mu_{S}$ in $\mathrm{J}(D)$, then $p \mu_{S}=p_{i}$, for some $1 \leq i \leq m$, and $q \mu_{S}=q_{i}^{j}$, for some $1 \leq j \leq k_{i}$, so $\Theta(\mathfrak{p}) \succ \Theta(\mathfrak{q})$ was guaranteed in $A_{i}$.

To establish that the size of $S$ is $O\left(n^{3}\right)$, we give a very crude upper bound for $|S|$. $2 n^{2}+1$ is an upper bound for $\left|I_{i}\right|, 1 \leq i \leq m$, so $3\left(2 n^{2}+1\right)$ is an upper bound 


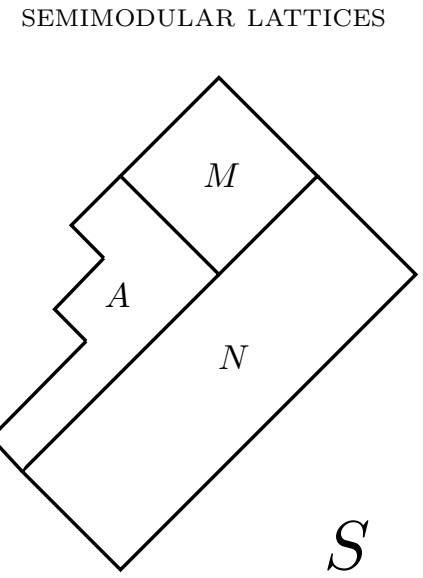

Figure 7

for $\left|A_{i}\right|$ and $3\left(2 n^{2}+1\right) n$ is an upper bound for $|A|$. Since $\left|D_{A}\right| \leq n+1$, we get the upper bound $(n+1)^{2}+n+1$ for $|M|$. Finally, $\left|I_{B}\right| \leq 2 n^{2}+1+n+1=2 n^{2}+n+2$, so $|N| \leq 2\left(2 n^{2}+n+2\right)(n+1)$. Therefore,

$$
3\left(2 n^{2}+1\right) n+(n+1)^{2}+n+1+2\left(2 n^{2}+n+2\right)(n+1)
$$

is an upper bound for $S$ and it is a cubic polynomial in $n$. This completes the proof of the Theorem.

It is not difficult to find better upper bounds for $|S|$; for instance,

$$
|S| \leq 3 n^{3}+2 n^{2}-7 n+4
$$

\section{REFERENCES}

[1] R. P. Dilworth, The Dilworth theorems. Selected papers of Robert P. Dilworth. Edited by Kenneth P. Bogart, Ralph Freese and Joseph P. S. Kung. Contemporary Mathematicians. Birkhäuser Boston, Inc., Boston, MA, 1990.

[2] G. Grätzer, General Lattice Theory, Pure and Applied Mathematics Series, Academic Press, New York; Mathematische Reihe, Band 52, Birkhäuser Verlag, Basel; Akademie Verlag, Berlin, 1978.

[3] G. Grätzer and H. Lakser, Homomorphisms of distributive lattices as restrictions of congruences, Canad. J. Math. 38 (1986), 1122-1134.

[4] Congruence lattices of planar lattices, Acta Math. Hungar. 60 (1992), 251-268.

[5] G. Grätzer, H. Lakser, and E. T. Schmidt, Congruence lattices of small planar lattices, Proc. Amer. Math. Soc. 123 (1995), 2619-2623.

[6] G. Grätzer, I. Rival, and N. Zaguia, Small representations of finite distributive lattices as congruence lattices, Proc. Amer. Math. Soc. 123 (1995), 1959-1961.

[7] G. Grätzer and E. T. Schmidt, On congruence lattices of lattices, Acta Math. Acad. Sci. Hungar. 13 (1962), 179-185.

[8] - Congruence-preserving extensions of finite lattices to sectionally complemented lattices, submitted to Proc. Amer. Math. Soc.

[9] $\ldots$, Congruence lattices of finite semimodular lattices, Abstracts Amer. Math. Soc. 97T06-56. 
Department of Mathematics, University of Manitoba, Winnipeg, Man. R3T 2N2, CANADA

E-mail address, G. Grätzer: gratzer@cc.umanitoba.ca

URL: http://www.maths. umanitoba.ca/homepages/gratzer.html/

E-mail address, H. Lakser: hlakser@cc.umanitoba.ca

Mathematical Institute of the Technical University of Budapest, Müegyetem RKP. 3, H-1521 Budapest, Hungary

E-mail address, E. T. Schmidt: schmidt@euromath.vma.bme.hu 